\title{
Stream-level Latency Evaluation for Simultaneous Machine Translation
}

\author{
Javier Iranzo-Sánchez and Jorge Civera and Alfons Juan \\ Machine Learning and Language Processing Group \\ Valencian Research Institute for Artificial Intelligence \\ Universitat Politècnica de València \\ Camí de Vera s/n, 46022 València, Spain \\ \{jairsan, jorcisai, ajuanci\} @vrain.upv.es
}

\begin{abstract}
Simultaneous machine translation has recently gained traction thanks to significant quality improvements and the advent of streaming applications. Simultaneous translation systems need to find a trade-off between translation quality and response time, and with this purpose multiple latency measures have been proposed. However, latency evaluations for simultaneous translation are estimated at the sentence level, not taking into account the sequential nature of a streaming scenario. Indeed, these sentence-level latency measures are not well suited for continuous stream translation, resulting in figures that are not coherent with the simultaneous translation policy of the system being assessed. This work proposes a stream-level adaptation of the current latency measures based on a re-segmentation approach applied to the output translation, that is successfully evaluated on streaming conditions for a reference IWSLT task.
\end{abstract}

\section{Introduction}

Simultaneous speech translation systems just started to become available (Bahar et al., 2020; Elbayad et al., 2020b; Han et al., 2020; Pham et al., 2020) thanks to recent developments in streaming automatic speech recognition and simultaneous machine translation. These systems seamlessly translate a continuous audio stream under real-time latency constraints. However, current translation latency evaluations (Ansari et al., 2020) are still performed at the sentence-level based on the conventional measures, Average Proportion (AP) (Cho and Esipova, 2016), Average Lagging (AL) (Ma et al., 2019) and Differentiable Average Lagging (DAL) (Cherry and Foster, 2019). These measures compute the translation latency for each sentence independently without taking into account possible interactions that lead to accumulated delays in a real-world streaming scenario. Additionally, the current measures cannot be used by systems that do not use explicit sentence-level segmentation (Schneider and Waibel, 2020).

In this work, we first revisit the conventional translation latency measures in Section 2 to motivate their adaptation to the streaming scenario in Section 3. Then, these adapted latency measures are computed and reported on an IWSLT task in Section 4. Finally, conclusions and future work are presented in Section 5.

\section{Related work}

Current latency measures for simultaneous translation can be characterised as a normalisation of the number of read-write word operations required to generate a translation $\boldsymbol{y}$ from a source sentence $\boldsymbol{x}$

$$
L(\boldsymbol{x}, \boldsymbol{y})=\frac{1}{Z(\boldsymbol{x}, \boldsymbol{y})} \sum_{i} C_{i}(\boldsymbol{x}, \boldsymbol{y})
$$

with $Z$ being a normalisation function, $i$ an index over the target positions and $C_{i}$ a cost function for each target position $i$. Depending on the latency measure, $C_{i}$ is defined as

$$
C_{i}(\boldsymbol{x}, \boldsymbol{y})= \begin{cases}g(i) & \mathrm{AP} \\ g(i)-\frac{i-1}{\gamma} & \mathrm{AL} \\ g^{\prime}(i)-\frac{i-1}{\gamma} & \mathrm{DAL}\end{cases}
$$

with

$$
g^{\prime}(i)=\max \left\{\begin{array}{l}
g(i) \\
g^{\prime}(i-1)+\frac{1}{\gamma}
\end{array}\right.
$$

where $g(i)$ is the number of source tokens read when a token is written at position $i$ and $\gamma$ is targetto-source length ratio $\frac{|\boldsymbol{y}|}{|\boldsymbol{x}|}$. Note that the AP cost function considers the absolute number of source tokens that has been read to output the $i$-th word, while AL and DAL cost functions account for the number of source words the model lags behind a wait- 0 oracle. This oracle simply accumulates a 
uniform distribution of source words over target positions according to the ratio $\frac{1}{\gamma}$. In the case of DAL, the recurrent definition of $g^{\prime}(i)$ guarantees that the most expensive read-write operation is considered.

On the other hand, the normalisation function $Z$ depends on the measure according to

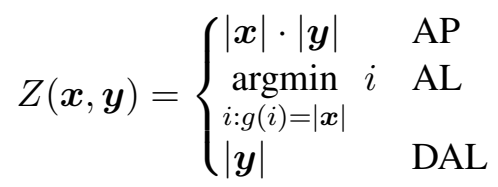

The term in AP normalises the sum over the target sentence of absolute source tokens, while AL and DAL does over the number of target positions, which in the case of AL is limited to those target positions reading new source tokens. Indeed, the normalization term of AL is referred to as $\tau$. The sentence-level latency measures just described are reported as an average value over an evaluation set of multiple sentence pairs, each one evaluated independently from the others.

However, the latency evaluation of a continuous paired stream of sentences has not received much attention, with the exception of the strategy proposed by (Schneider and Waibel, 2020). This evaluation strategy considers the straightforward approach of concatenating all sentences into a single source/target pair in order to compute the corresponding latency measure. Next section outlines some drawbacks of this strategy (hereafter Concat1) to motivate the discussion on how the current sentence-level latency measures could be adapted to the streaming scenario.

\section{Stream-level evaluation}

Let us consider the translation of a stream of two sentences, the first sentence has two input and two output tokens, while the second one has two input and four output tokens with ratios $\gamma_{1}=1$ and $\gamma_{2}=2$, respectively. The translation process is performed with a sentence-based wait-k system with catch-up characterised by a function $g(i)=$ $\left\lfloor k+\frac{i-1}{\gamma}\right\rfloor$ with $k=1$.

Table 1 compares the computation of the latency measures for the Concat-1 strategy (top) with the conventional strategy that considers independent sentences (bottom). Note that the translation process has only been carried out once, but both strategies are just interpreting the results differently as first denoted by their $i$ and $g(i)$ values. The wait- 0 oracle $\frac{i-1}{\gamma}$ of Concat- 1 , with a single global $\gamma=\frac{3}{2}$
Table 1: Comparison of the latency metric computation between the Concat-1 (top) and the conventional sentence-level (bottom) strategy when using a wait-1 system.

\begin{tabular}{|c|c|c|c|c|c|c|c|c|c|}
\hline & & & & & & & & & $L$ \\
\hline \multirow{6}{*}{ 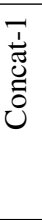 } & & $i$ & 1 & 2 & 3 & 4 & 5 & 6 & \\
\hline & & $g(i)$ & 1 & 2 & 3 & 3 & 4 & 4 & \\
\hline & & $\frac{i-1}{\gamma}$ & 0 & 0.6 & 1.3 & 2.0 & 2.6 & 3.3 & \\
\hline & \multirow{3}{*}{$C_{i}$} & AP & 1 & 2 & 3 & 3 & 4 & 4 & 0.7 \\
\hline & & AL & 1 & 1.3 & 1.6 & 1 & 1.3 & - & 1.2 \\
\hline & & DAL & 1 & 1.3 & 1.6 & 1.6 & 1.6 & 1.6 & 1.5 \\
\hline \multirow{3}{*}{ 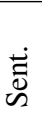 } & & $i$ & 1 & 2 & 1 & 2 & 3 & 4 & \\
\hline & & $g(i)$ & 1 & 2 & 1 & 1 & 2 & 2 & \\
\hline & & $\frac{i-1}{\gamma}$ & 0.0 & 1.0 & 0.0 & 0.5 & 1.0 & 1.5 & \\
\hline \multirow{3}{*}{ ટ્ઢ } & & AP & 1 & 2 & 1 & 1 & 2 & 2 & 0.8 \\
\hline & $C_{i}$ & AL & 1 & 1 & 1 & 0.5 & 1 & - & 0.9 \\
\hline & & DAL & 1 & 1 & 1 & 1 & 1 & 1 & 1.0 \\
\hline
\end{tabular}

underestimates the actual writing rate, and the system accumulates more delay than in the evaluation strategy of independent sentences, which uses a sentence-level estimation for $\gamma$.

These differences in results are magnified when computing latencies on a real streaming evaluation set. On the one hand, AL and DAL tend to obtain scores that do not reflect the real behaviour of the system when using a Concat-1 strategy with a single global $\gamma$, since the source-target length ratio varies wildly between different sentences. Therefore, the wait- 0 oracle will sometimes overestimate the actual writing rate, and sometimes it will underestimate it. Moreover, the definition of DAL keeps the system from recovering from previously incurred delays, and therefore, every time the writing rate is underestimated, the system falls further and further behind the wait- 0 oracle. On the other hand, AP turns out to be little informative when the stream is long enough, since AP always tends to be 0.5 because the delay incurred by a system with a reasonable $k$ is always negligible compared with the total source length.

The accuracy of AL and DAL could be improved if sentence-level estimations for $\gamma$ would be available somehow in a streaming scenario. With the availability of these estimations in mind, we formulate a streaming version of the cost functions in Eq. 2 based on a global $G(i)$ function, which returns the number of source tokens (including those from previous sentences) that have been read as in 
Table 2: Estimation of stream-level latencies measures on the same example proposed in Table 1.

\begin{tabular}{|c|c|c|c|c|c|c|c|c|}
\hline & $i$ & & 2 & & 2 & 3 & 4 & $L$ \\
\hline \multirow[b]{5}{*}{$C_{i}$} & $G\left(i+\left|\boldsymbol{u}_{1}^{n-1}\right|\right)$ & 1 & 2 & 3 & 3 & 4 & 4 & \\
\hline & $g_{n}(i)$ & 1 & 2 & 1 & 1 & 2 & 2 & \\
\hline & $\frac{i-1}{\gamma_{n}}$ & 0.0 & 1.0 & 0.0 & 0.5 & 1.0 & 1.5 & \\
\hline & AP & 1 & 2 & 1 & 1 & 2 & 2 & 0.8 \\
\hline & $\mathrm{AL}$ & 1 & 1 & 1 & 0.5 & 1 & - & 0.9 \\
\hline & DAL & 1 & 1 & 1 & 1 & 1 & 1 & 1.0 \\
\hline
\end{tabular}

the Concat-1 strategy:

$$
C_{i}\left(\boldsymbol{x}_{n}, \boldsymbol{y}_{n}\right)= \begin{cases}g_{n}(i) & \mathrm{AP} \\ g_{n}(i)-\frac{i-1}{\gamma_{n}} & \mathrm{AL} \\ g_{n}^{\prime}(i)-\frac{i-1}{\gamma_{n}} & \mathrm{DAL}\end{cases}
$$

with $g_{n}^{\prime}(i)$ defined as

$$
\max \left\{\begin{array}{l}
g_{n}(i) \\
\begin{cases}g_{n-1}^{\prime}\left(\left|\boldsymbol{x}_{n-1}\right|\right)+\frac{1}{\gamma_{n-1}} & i=1 \\
g_{n}^{\prime}(i-1)+\frac{1}{\gamma_{n}} & i>1\end{cases}
\end{array}\right.
$$

where $g_{n}(i)=G\left(i+\left|\boldsymbol{y}_{1}^{n-1}\right|\right)-\left|\boldsymbol{x}_{1}^{n-1}\right|$. Thus, the global delay is converted to a local representation so that it can be compared with the local sentence oracle.

Table 2 shows the computation of the streamlevel latency measures as proposed in Eq. 5 for the same example calculated in Table 1. As observed, unlike with the Concat-1 strategy, we obtain the same results as in the conventional sentencelevel estimation, while at the same time we keep the property that previous delays affect future sentences by basing our computations on the global delay $G(i)$.

If we use a segmentation-free model whose output is a single text stream, stream-level latency measures can be still computed by re-segmenting the output into sentence-like units (chunks). Formally, a segmenter takes an input stream $Y$ and a set of reference sentences to compute a re-segmentation $\hat{\boldsymbol{y}}_{1}^{N}$ of $Y$. Once the re-segmentation is obtained, stream-level latency measures are estimated by considering paired input-output segments $\left(\boldsymbol{x}_{n}, \hat{\boldsymbol{y}}_{n}\right)$. In our case, we re-segment by minimizing the edit distance between the stream hypothesis and the reference translations, analogously to the translation quality evaluation widely-used in speech translation (Matusov et al., 2005). Likewise, we can resegment the output to compute latency measures if our system uses a different segmentation than the reference.

Moreover, stream-level AL and DAL measures computed for a wait- $k$ system are coherently close to $k$ with two caveats. First, there can be deviation from the theoretical value of $k$ due to a inaccurate estimation of the writing rate. Given that the wait $k$ policy uses a fixed $\gamma$, there will be some sentences in which this results in lower or higher writing rates than desirable. This is a feature inherent to the fixed policy itself. Second, a deviation could also occur due to re-segmentation errors. For instance, a word that is part of the translation of the $n$-th segment can be wrongly included into the previous $n$-1-th segment causing an increase of the latency. Both sources of latency are illustrated in Figure 1.

These two caveats given the definition of DAL imply that a system can never recover from previous delays, which might be an acceptable solution when computing latency measures at the sentence level, but it seems too strict and unrealistic when computing latency measures for streams comprising tens of thousands of words. To alleviate this problem, we propose to multiply the cost of a write operation $\frac{1}{\gamma_{n}}$ in $g_{n}^{\prime}(i)$ by a scaling factor $s \in[0,1]$. In practice, for values of $s$ close to 1 , this means that the write operation costs slightly less for the real system than for the oracle. We believe this is an acceptable practical solution given that there are many ways that this could be achieved in realworld tasks, such as rendering subtitles slightly faster or, in the case of cascade speech-to-speech, slight reducing the duration of TTS segments or increasing the playback speed. Finally, the scaling factor $s$ can be also understood as a hyperparameter that bridges the gap between $\operatorname{AL}(s=0)$ and DAL $(s=1)$ and it can be adjusted depending on the actual writing cost of the translation task.

\section{Experiments}

The stream-level latency measures proposed in Section 3 are now computed and evaluated on the IWSLT 2010 German-English dev set (Paul et al., 2010). To simulate a streaming scenario, all source sentences are concatenated into a single input stream. Then, they are segmented into sentences and translated with a wait- $k$ fixed policy. As a result, it is expected that a well-behaved latency measure should rank the systems by increasing order of $k$.

Our streaming simultaneous translation sys- 


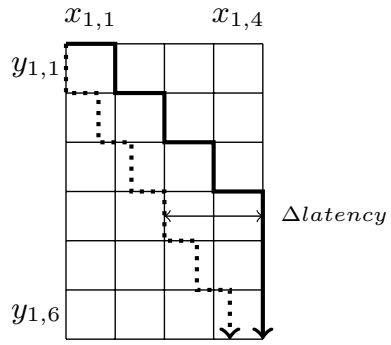

(a)

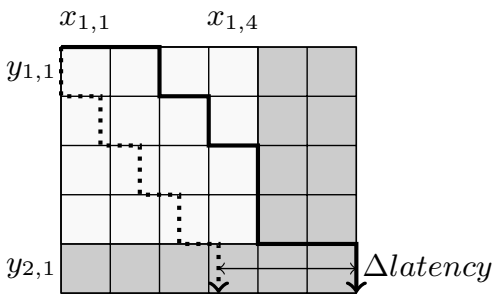

(b)

Figure 1: The examples shown above illustrate how a model which follows a wait- $k$ policy can obtain AL/DAL values that differ from $k$. The bold lines show the behaviour of the model, the dotted lines show the oracle policy. Left: writing rate error with $k=1$; the model uses $\hat{\gamma}=1$, but the actual value is $\gamma=1.5$. Right: segmentation error with $k=2$; the first translated word of the second sentence is wrongly assigned to the first sentence during resegmentation, i.e. $\hat{y}_{1}=\left(y_{1,1}, y_{1,2}, y_{1,3}, y_{1,4}, y_{2,1}\right)$.

tem is based on a direct segmentation (DS) model (Iranzo-Sánchez et al., 2020) followed by a Transformer BASE model (Vaswani et al., 2017) trained with the multi- $k$ approach (Elbayad et al., 2020a). The DS model was trained on TED talks (Cettolo et al., 2012) with a future window of length 0 and history size of 10 , while the translation model was trained on the IWSLT 2020 GermanEnglish data (Ansari et al., 2020). This system, which we will refer to as Real, uses catch-up with $\gamma=1.24$. In addition to the Real system, three experimental setups based on different oracles are considered:

- In. Seg.: The input segmentation provided by the DS model is replaced by the reference segmentation to gauge segmentation errors.

- Out. Seg.: The reference segmentation is used to link each translation with its corresponding source sentence, therefore avoiding the need of re-segmentation by minimum edit distance.

- Policy: The translation model is replaced by an oracle model that outputs the reference translation with the appropriate writing rate for each sentence to account for errors due to a global $\gamma$.

AL (Table 3) and DAL (Table 4) have been computed using the Concat-1 approach, to serve as a baseline for the developed measures. These results confirm the problems of the Concat-1 approach, which have been identified and discussed on Section 3. AP results have been excluded from the tables, as no matter which setup is used, the computed AP is always 0.5. Likewise, the obtained AL and DAL values offer little insight about the latency
Table 3: Stream-level AL as a function of $k$, computed using the Concat-1 approach on the IWSLT 2010 German-English dev set.

\begin{tabular}{l||rrrrr} 
System & 1 & 2 & 3 & 4 & 5 \\
\hline Real & -9.7 & -12.0 & -45.2 & -23.7 & -8.5 \\
$\quad$ In. Seg. & -42.9 & -29.0 & 17.4 & -10.1 & 25.5 \\
+ Policy & 14.2 & 15.1 & 16.0 & 16.8 & 17.6
\end{tabular}

behaviour of the model. These results are not only uninterpretable, but they also alter the ranking of the models. This could be specially worrisome if the Concat-1 approach was used to compare systems with adaptative policies that lack a explicit latency control such as $k$, as it might be harder to detect wheter the incoherent results are due to the adaptative policy or the latency measure itself. The only setup which returns the correct ranking is the one using the In. Seg. and Policy Oracles, but the latency results do not reflect the real behaviour of the model. The full AL and DAL results, for values up to $k=10$ are reported in the appendix.

Table 4: Stream-level DAL as a function of $k$, computed using the Concat-1 approach on the IWSLT 2010 German-English dev set.

\begin{tabular}{l||rrrrr} 
System & 1 & 2 & 3 & 4 & 5 \\
\hline Real & 15.0 & 11.0 & 17.4 & 11.3 & 20.3 \\
+In. Seg. & 4.5 & 8.5 & 37.1 & 24.6 & 52.3 \\
+ Policy & 85.8 & 86.7 & 87.7 & 88.7 & 89.7
\end{tabular}

Now that we have experimentally shown that the Concat- 1 approach is unable to properly compute latencies, we move onto computing the streamadapted version of the latency measures. The computation of stream-level AP (left), AL (center) and, 

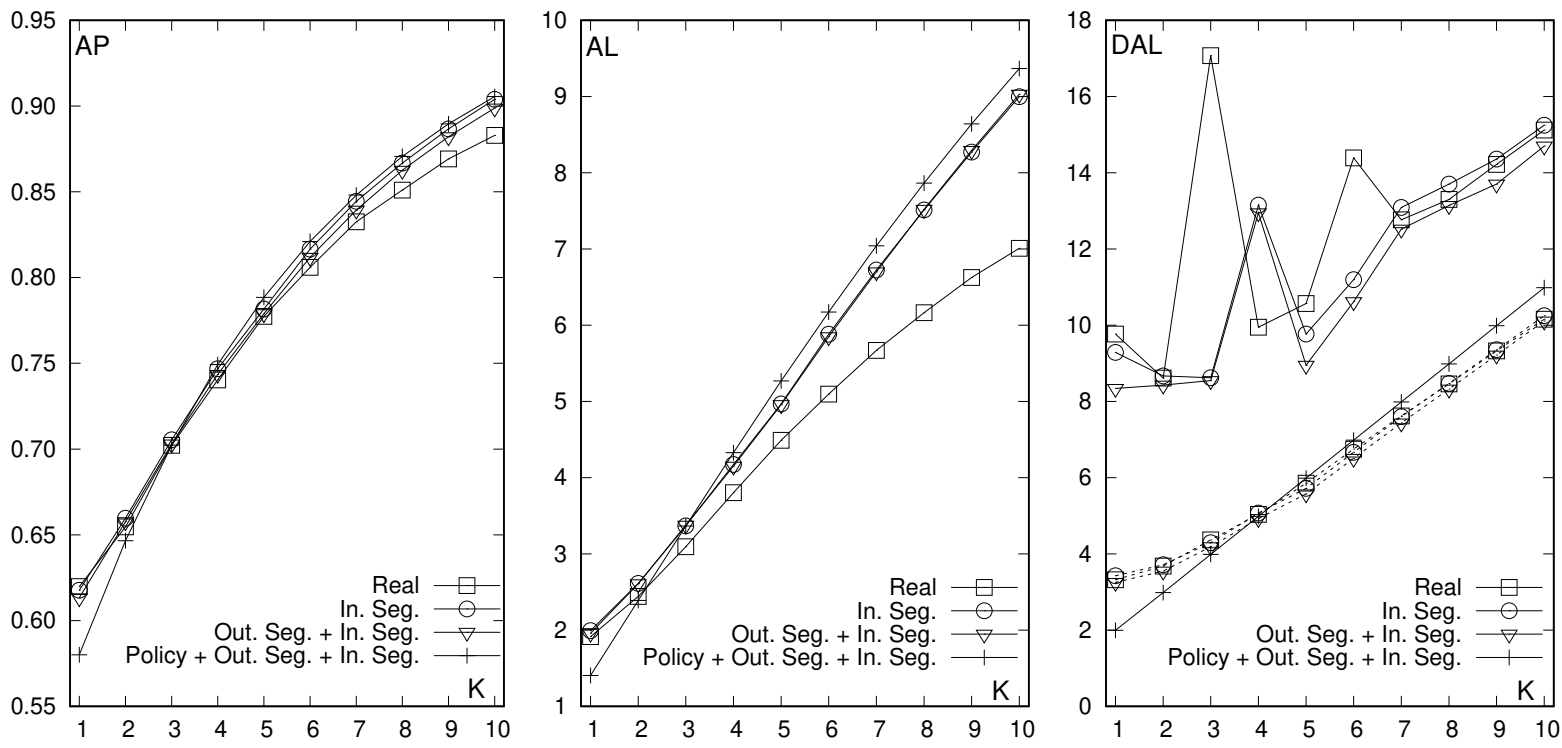

Figure 2: Stream-level AP (left), AL (center) and DAL (right) with $s=1.0$ and $s=0.95$ (dashed lines) as a function of $k$ in the multi- $k$ approach for four experimental setups on the IWSLT 2010 German-English dev set.

DAL (right) with $s=1.0$ and $s=0.95$ (dashed lines) as a function of $k$ in the multi- $k$ approach are shown in Figure 2. The behaviour of AP and AL is that expected for the four experimental setups defined above, but the conventional DAL measure $(s=1.0)$ abruptly suffers the effect of not being able to recover from accumulated delays due to the cost of write operations. In contrast, DAL with $s=0.95$ exhibits a smooth interpretable behaviour as a result of compensating for re-segmentation errors. Moreover, the gap between "In. Seg." and "In. Seg. + Out. Seg." is not significant, therefore we believe that, if the translation quality is good enough, the automatic re-segmentation process is an acceptable way of computing stream-level latencies. Lastly, as expected, if we use an oracle system that outputs the reference translation with the appropriate writing rate for each sentence ("Policy + In. Seg. + Out. Seg."), the obtained AL and DAL values are very close to the theoretical value $k$. If we compute DAL using $s=0.95$, we obtain similar values without the need of using any oracle, while accounting for the additional cost of write operations.

Thus, unlike the Concat-1 approach, our streamlevel approach is highly effective for providing interpretable and accurate latency measures.

\section{Conclusions}

In this work, an adaptation of the current latency measures to a streaming setup is proposed moti- vated by the lack of interpretability of sentencelevel latency measures in this setup.

This adaptation basically consists in the modification of the conventional latency measures to move from a sentence-level evaluation based on a local delay function to a stream-level estimation by using a global delay function that keeps track of delays across the whole translation process. At the same time, a re-segmentation approach has been proposed to compute these latency measures on any arbitrary segmentation of the input stream used by the translation model. The resulting measures are highly interpretable and coherent accounting for the actual behaviour of the simultaneous translation system in a real streaming scenario.

\section{Acknowledgements}

The research leading to these results has received funding from the European Union's Horizon 2020 research and innovation program under grant agreement no. 761758 (X5Gon) and 952215 (TAILOR) and Erasmus+ Education program under grant agreement no. 20-226-093604-SCH; the Government of Spain's research project Multisub, ref. RTI2018-094879-B-I00 (MCIU/AEI/FEDER,EU) and FPU scholarships FPU18/04135; and the Generalitat Valenciana's research project Classroom Activity Recognition, ref. PROMETEO/2019/111. 


\section{References}

Ebrahim Ansari, Amittai Axelrod, Nguyen Bach, Ondřej Bojar, Roldano Cattoni, Fahim Dalvi, Nadir Durrani, Marcello Federico, Christian Federmann, Jiatao Gu, Fei Huang, Kevin Knight, Xutai Ma, Ajay Nagesh, Matteo Negri, Jan Niehues, Juan Pino, Elizabeth Salesky, Xing Shi, Sebastian Stüker, Marco Turchi, Alexander Waibel, and Changhan Wang. 2020. FINDINGS OF THE IWSLT 2020 EVALUATION CAMPAIGN. In Proc. of IWSLT, pages 1-34, Online. ACL.

Parnia Bahar, Patrick Wilken, Tamer Alkhouli, Andreas Guta, Pavel Golik, Evgeny Matusov, and Christian Herold. 2020. Start-Before-End and Endto-End: Neural Speech Translation by AppTek and RWTH Aachen University. In Proc. of IWSLT, pages 44-54. ACL.

Mauro Cettolo, Christian Girardi, and Marcello Federico. 2012. $\mathrm{WIT}^{3}$ : Web Inventory of Transcribed and Translated Talks. In Proc. of EAMT.

Colin Cherry and George Foster. 2019. Thinking slow about latency evaluation for simultaneous machine translation. arXiv preprint arXiv:1906.00048.

Kyunghyun Cho and Masha Esipova. 2016. Can neural machine translation do simultaneous translation? arXiv preprint arXiv:1606.02012.

Maha Elbayad, Laurent Besacier, and Jakob Verbeek. 2020a. Efficient Wait-k Models for Simultaneous Machine Translation. In Proc. of Interspeech, pages 1461-1465.

Maha Elbayad, Ha Nguyen, Fethi Bougares, Natalia Tomashenko, Antoine Caubrière, Benjamin Lecouteux, Yannick Estève, and Laurent Besacier. 2020b. ON-TRAC consortium for end-to-end and simultaneous speech translation challenge tasks at IWSLT 2020. In Proc. of IWSLT, pages 35-43. ACL.

Hou Jeung Han, Mohd Abbas Zaidi, Sathish Reddy Indurthi, Nikhil Kumar Lakumarapu, Beomseok Lee, and Sangha Kim. 2020. End-to-end simultaneous translation system for IWSLT2020 using modality agnostic meta-learning. In Proc. of IWSLT, pages 62-68. ACL.

Javier Iranzo-Sánchez, Adrià Giménez, Joan Albert Silvestre-Cerdà, Pau Baquero, Jorge Civera, and Alfons Juan. 2020. Direct Segmentation Models for Streaming Speech Translation. In Proc. of EMNLP, pages 2599-2611. ACL.

Mingbo Ma, Liang Huang, Hao Xiong, Renjie Zheng, Kaibo Liu, Baigong Zheng, Chuanqiang Zhang, Zhongjun He, Hairong Liu, Xing Li, Hua $\mathrm{Wu}$, and Haifeng Wang. 2019. STACL: Simultaneous translation with implicit anticipation and controllable latency using prefix-to-prefix framework. In Proc. of $A C L$, pages 3025-3036. ACL.
Evgeny Matusov, Gregor Leusch, Oliver Bender, and Hermann Ney. 2005. Evaluating machine translation output with automatic sentence segmentation. In Proc. of IWSLT. ISCA.

Michael Paul, Marcello Federico, and Sebastian Stüker. 2010. Overview of the iwslt 2010 evaluation campaign. In Proc. of IWSLT, pages 3-27. ISCA.

Ngoc-Quan Pham, Felix Schneider, Tuan-Nam Nguyen, Thanh-Le Ha, Thai Son Nguyen, Maximilian Awiszus, Sebastian Stüker, and Alexander Waibel. 2020. KIT's IWSLT 2020 SLT translation system. In Proc. of IWSLT, pages 55-61. ACL.

Felix Schneider and Alexander Waibel. 2020. Towards stream translation: Adaptive computation time for simultaneous machine translation. In Proc. of IWSLT, pages 228-236. ACL.

Ashish Vaswani, Noam Shazeer, Niki Parmar, Jakob Uszkoreit, Llion Jones, Aidan N. Gomez, Lukasz Kaiser, and Illia Polosukhin. 2017. Attention is all you need. In Proc. of NIPS, pages 5998-6008.

\section{A Reproducibility of proposed measures}

The code for the proposed latency measures, as well as all the translations have been published 1 . A script is included to reproduce the results reported in the paper. The full results for the Concat-1 method are reported on Tables 5 and 6

\section{B MT System}

Table 7 lists the corpus that were selected for training out of the IWSLT 2020 allowed data ${ }^{2}$.

The multi- $k$ system has been trained with the official implementation ${ }^{3}$. The model was trained for $0.5 \mathrm{M}$ steps on a machine with 2 2080Ti GPUs, which took 6 days. The following command was used to train it:

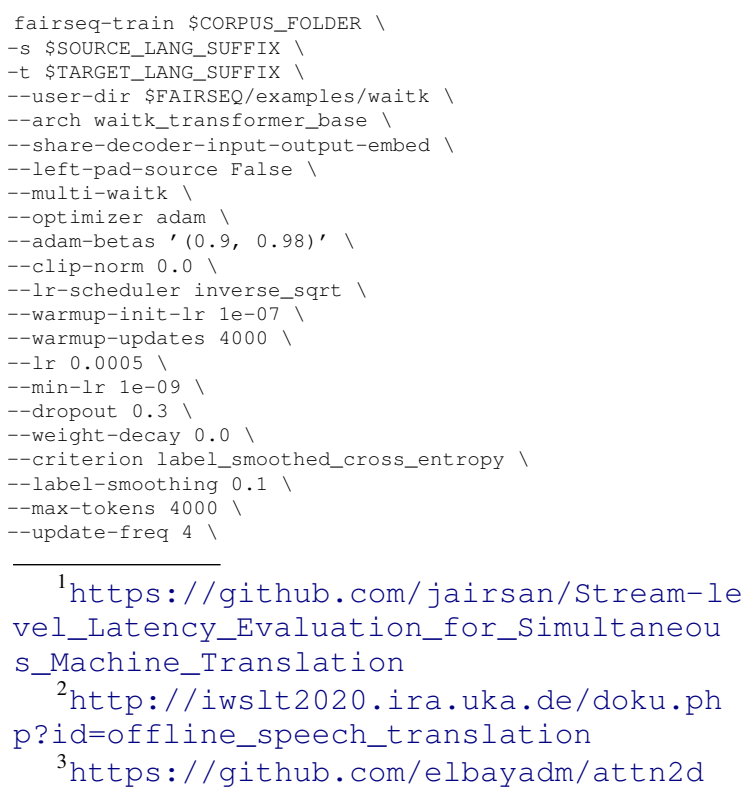


Table 5: Stream-level AL as a function of $k$, computed using the Concat-1 approach on the IWSLT 2010 GermanEnglish dev set.

\begin{tabular}{l||rrrrrrrrrr} 
System & 1 & 2 & 3 & 4 & 5 & 6 & 7 & 8 & 9 & 10 \\
\hline Real & -9.7 & -12.0 & -45.2 & -23.7 & -8.5 & -4.4 & -17.4 & -13.6 & -14.2 & -12.2 \\
$\quad$ In-Seg Oracle & -42.9 & -29.0 & 17.4 & -10.1 & 25.5 & 3.8 & 9.7 & 5.3 & 2.7 & 4.7 \\
$\quad$ + Policy Oracle & 14.2 & 15.1 & 16.0 & 16.8 & 17.6 & 18.2 & 18.9 & 19.5 & 20.1 & 20.6
\end{tabular}

Table 6: Stream-level DAL as a function of $k$, computed using the Concat-1 approach on the IWSLT 2010 GermanEnglish dev set.

\begin{tabular}{l||rrrrrrrrrr} 
System & 1 & 2 & 3 & 4 & 5 & 6 & 7 & 8 & 9 & 10 \\
\hline Real & 15.0 & 11.0 & 17.4 & 11.3 & 20.3 & 25.1 & 11.3 & 14.4 & 17.7 & 17.9 \\
$\quad$ In-Seg Oracle & 4.5 & 8.5 & 37.1 & 24.6 & 52.3 & 27.8 & 21.5 & 33.9 & 31.2 & 31.8 \\
$\quad$ + Policy Oracle & 85.8 & 86.7 & 87.7 & 88.7 & 89.7 & 90.7 & 91.7 & 92.7 & 93.6 & 94.6
\end{tabular}

Table 7: Corpus used for MT model training

\begin{tabular}{l||rrr}
\multicolumn{1}{c||}{} & \multicolumn{3}{c}{ tokens(M) } \\
Corpus & sentences(M) & German & English \\
\hline News Commentary & 0.3 & 7.4 & 7.2 \\
WikiTitles & 1.3 & 2.7 & 3.1 \\
Europarl & 1.8 & 42.5 & 45.5 \\
Rapid & 1.5 & 26.0 & 26.9 \\
MuST-C & 0.2 & 3.9 & 4.2 \\
Ted & 0.2 & 3.3 & 3.6 \\
LibriVox & 0.1 & 0.9 & 1.1 \\
Paracrawl & 31.4 & 465.2 & 502.9
\end{tabular}

--save-dir \$MODEL_OUTPUT_FOLDER ।

--no-progress-bar ।

- log-interval 100

--save-interval-updates 10000

--save-interval-updates 1000
--keep-interval-updates $20 \backslash$

--ddp-backend=no_c10d

$--\mathrm{fp} 16$

\section{Segmenter System}

The Direct Segmentation system has been trained with the official implementation ${ }^{4}$. The ted corpus was used as training data (See Table 7). The following command was used to train the segmenter system:

len=11

python3 train_text_model.py

--train_corpus train.ML\$len.WS\$window.txt

--dev_corpus dev.ML\$len.WS\$window.txt

-output_folder \$output_folder

--vocabulary \$corpus_folder/train.vocab.txt >

--checkpoint_interval 1 ।

--epochs 15

-rnn_layer_size 256

--embedding_size 256

--n_classes 2 ।

--batch_size 256 ।

-min_split_samples_batch_ratio 0.3 ।

-optimizer adam

- - Ir 0.0001 )

-Ir_schedule reduce_on_plateau ।

- - lr_reduce_patience 5

--dropout 0.3 ।

-model architecture ff-text

--feedforward_layers 2

-feedforward_size 128

-sample max len \$len $\backslash$

--sample_window_size \$window

${ }^{4}$ https://github.com/jairsan/Speech_Tra nslation_Segmenter 